\title{
Linking Additive Structures to Nanoparticle Properties
}

\author{
William R. Richmond, Gordon M. Parkinson, Franca Jones, Mark I. Ogden, \\ Allan Oliveira, Manijeh Reyhani, and Andrew L. Rohl \\ Nanochemistry Research Institute and A.J. Parker CRC for Hydrometallurgy, Curtin University of \\ Technology, GPO Box U1987, Perth 6845, Australia.
}

email: bill@power.curtin.edu.au

Keywords: nanoparticles, additives, crystal growth, phophonates, carboxylates.

\begin{abstract}
The effects of a series of polyphosphonate and poly-carboxylate additives have been investigated in the crystallization of various inorganic salts. Systematic variation of the additive structure has been used to provide insight into the dominant factors in additive-crystal interactions. The results obtained for barium sulfate and hematite $\left(\alpha-\mathrm{Fe}_{2} \mathrm{O}_{3}\right)$ show that the morphological effects do not necessarily follow the trend one might expect on the basis of the structural features of the additives. Molecular modeling, coupled with in-situ AFM imaging is being used to develop an approach that will allow more informed systematic design of crystal growth modifiers.
\end{abstract}

\section{Introduction}

Control of size and shape in the growth of nanoparticles and nanocomposites is elegantly demonstrated by nature in a number of biological processes in which macromolecules direct specific interactions with growing crystal surfaces via functional groups on proteins or other macromolecules. Similarly, in industrial processes, chemical additives are often employed to control the shape and size of crystalline products. They can also serve to disrupt the unwanted growth of crystalline material as scale on reactor and heat exchanger surfaces. In most cases the selection of such additives has been developed by empirical methods, and the mechanisms by which they work are poorly understood.

In the production of nanoparticles of specific morphology and composition, this empirical approach has already led to a wide range of developments, and in some cases very specific control has been achieved. The use of micelles and microemulsions as "nanoreactors" for example, has enabled very precise control of size in some systems [1]. Our ultimate goal however, is to be able to produce nanoparticles of specific morphological characteristics through the rational design of appropriate additives or macromolecular matrices. Achieving this goal will require a detailed understanding of how various functional groups interact with growing crystal surfaces under a wide range of conditions, and throughout a wide range of crystal systems. Our objective may be a long way off, but the initial steps towards it are being taken through the study of crystal growth under the influence of additives containing systematic variations in their structural design.

\section{Systematic variation of additive structure}

Our group has studied the effects of a series of poly-phosphonate and a series mixed polyphosphonate/carboxylate additives on the crystallization of various sparingly soluble salts. The structures of these additives are presented in Fig. 1. 

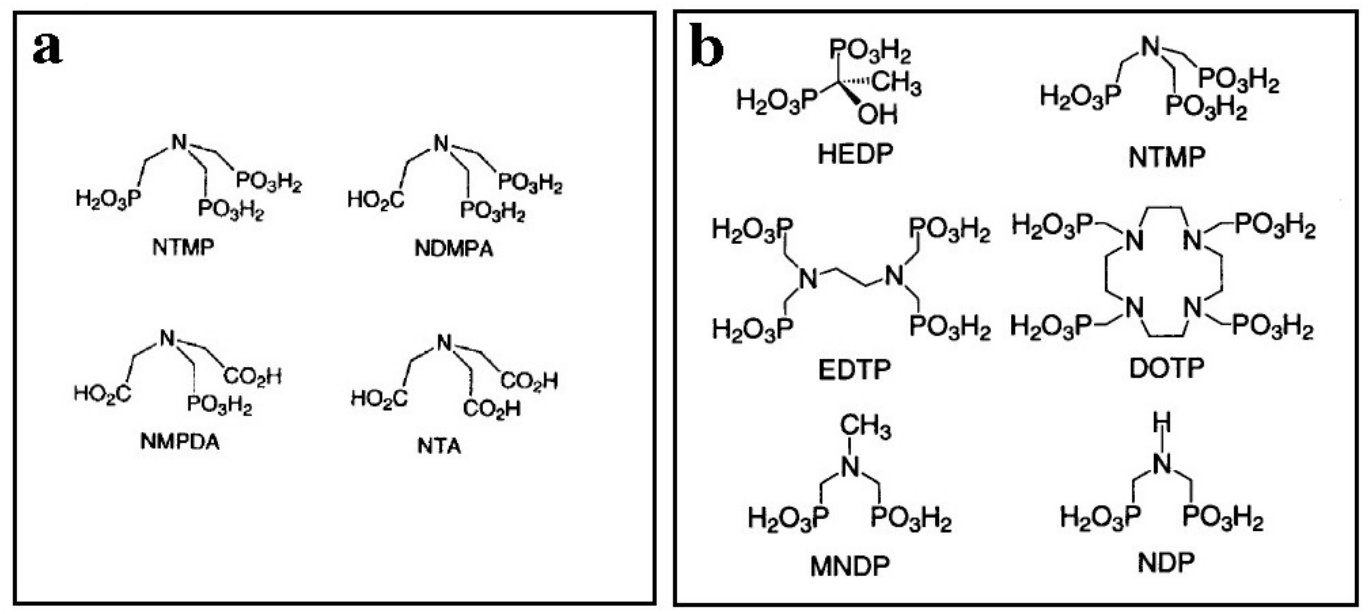

Fig. 1. Systematic variation of additive structures. a) A series of mixed phophonate/carboxylate additives with the same geometric arrangement of functional groups. b) A series of polyphosphonate additives with different numbers of phosphonate groups or different geometric arrangement of phosphonate groups. The abbreviated names of the additives are given.

The series of mixed phosphonate/carboxylate additives shown in Figure 1a were studied as crystal growth modifiers in the precipitation of barium sulfate [2]. The generally accepted view is that phosphonates are much more potent inhibitors of barium sulfate precipitation than the analogous carboxylates, and given that the geometry of the additives was the same in each case, it was anticipated that the efficacy of the additives would decrease as the number of phosphonate groups decreased. If we consider only the additives NTMP, NDMPA and NMPDA then the observed inhibition behaviour and crystal morphology changes followed the expected trend, but surprisingly, NTA was found to behave differently from the other additives [3]. As a growth inhibitor NTA outperformed both the mono- and di- phosphonate containing molecules at the same concentrations, and the morphology changes induced by this molecule were different from those effected by the other additive. These observations suggest that the mode of interaction of NTA with the crystal surface is different from that of the other additives.

The study of a systematic series of additive structures has also been applied to the synthesis of iron oxide nanoparticles, and as an example, the acid hydrolysis of ferric chloride in the presence of EDTP to produce hematite is presented in Fig. 2. In these experiments, inhibitory effects and morphology changes were investigated for a number of poly-phosphonate molecules having a varying number of functional groups, as well as different structural arrangements of the phosphonate moieties.

In this case, the additives were all found to induce the same morphology changes: the initially rhombic nanoparticles became truncated at the ends as the additive concentration was increased. High resolution TEM revealed that the additives were binding and inhibiting growth at the $(2 \overline{1} \overline{4})$ face of the crystals [4]. Studies of growth rates under the influence of the different additives revealed that the efficacy of these additives did not vary greatly in this system. The performance of the additives as inhibitors was evaluated by measuring de-supersaturation curves for iron, a process that involved regular sampling of a precipitation reaction followed by centrifugation and $\mathrm{Fe}$ analysis of the supernatant using AAS. It was found that there was no relationship between the number of phosphonate groups and the inhibitory effect of a given poly-phosphonate. Furthermore, there appeared to be no effect arising from the different structural arrangement of the groups. For example, DOTP and EDTP were found to have the same inhibitory effect at the same concentrations, and the inhibitory effect of a given concentration of EDTP equated to that effected by twice as much MNDP. Thus we conclude that the interaction of these molecules with the iron 
oxide surface involves, at most, two phosphonate functionalities, and the presence of additional phosphonate groups does not enhance their effect at this $\mathrm{pH}$.
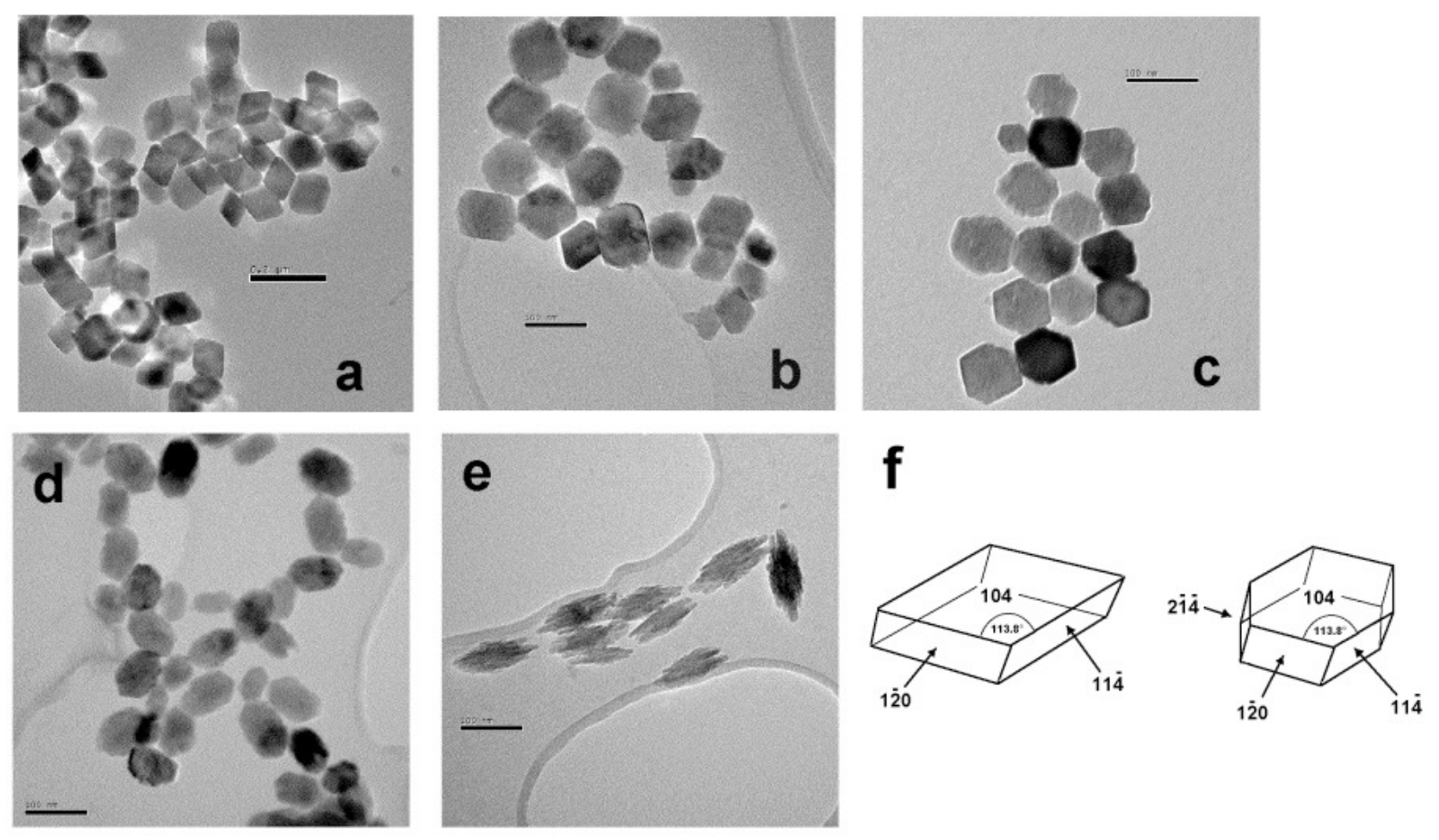

Fig. 2: Hematite nanoparticles formed in the presence of increasing amounts of EDTP. a) no additive, b) $[$ EDTP] $=0.5$ $\mathrm{ppm}, \mathrm{c})[\mathrm{EDTP}]=5 \mathrm{ppm}, \mathrm{d})[\mathrm{EDTP}]=10 \mathrm{ppm}, \mathrm{e})[\mathrm{EDTP}]=20 \mathrm{ppm}$. f) Schematic representation of hematite morphologies in the absence of additive (left) and in the presence of phosphonate (right). Size bar in a) $=200 \mathrm{~nm}$, all other size bars $=100 \mathrm{~nm}$.

\section{Molecular level study of additive-surface interactions}

While the examples described above have provided important information about the factors involved in morphology control for specific systems, they also serve to highlight the limitations of trying to understand how particle morphology and size can be controlled though design of additive structures. This has led us to seek ways of probing the nature of additive-surface interactions at the molecular level, through molecular modeling and Atomic Force Microscopy.

Molecular modeling of EDTP and DOTP on flat barium sulfate surfaces has shown that both additives will try to lattice-match the positions of sulfate (see Fig. 3 for the case of the (001) face) within the structure. According to replacement energy calculations they should both be good inhibitors; this is not, however, the experimental reality.

(a)

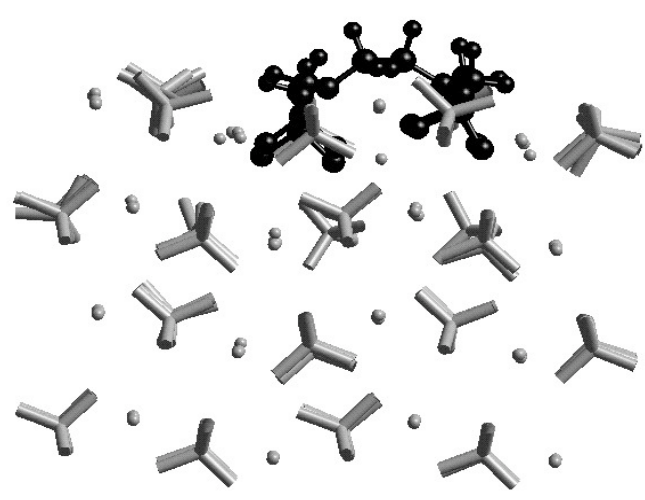

(b)

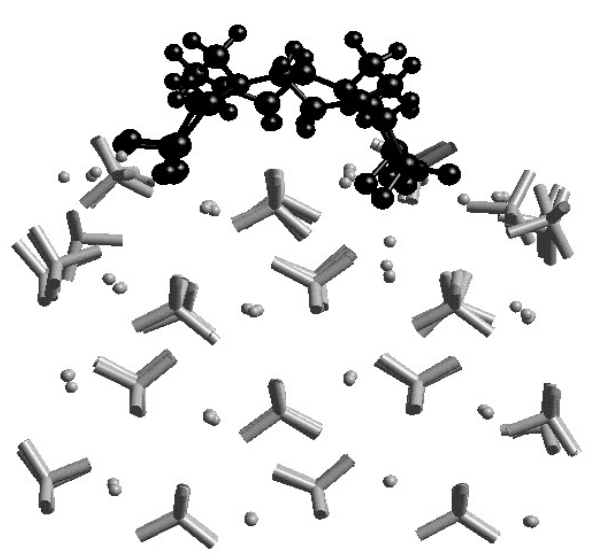

Fig. 3: (a) EDTP and (b) DOTP adsorbed on the flat (001) face of barium sulfate. Side view shown. 


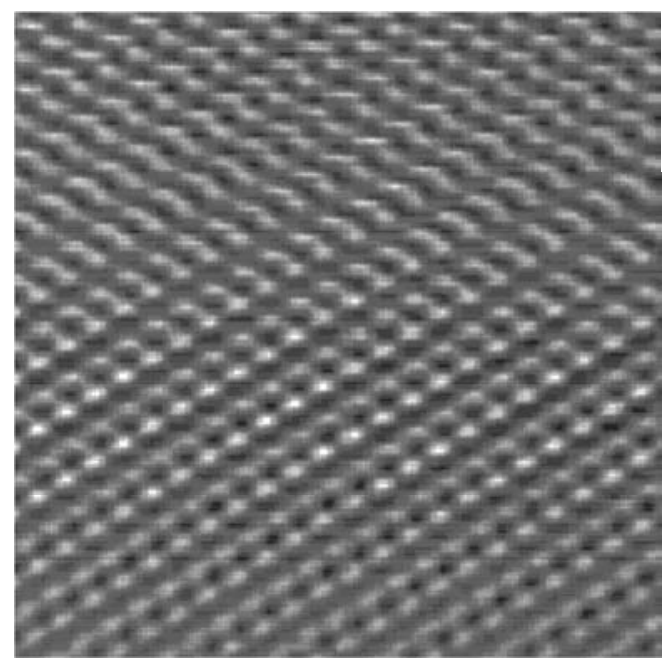

Fig. 4 : Atomic resolution AFM image of a calcite surface. The pairs of white dots in rows are pairs of oxygen atoms in carbonate groups at the surface.
Previous modeling on calcite [5] has shown that a possible source of this 'discrepancy' could be that adsorption of these additives onto flat surfaces is not the important criterion. Instead, the interaction of these additives with growth features such as steps or spirals may be a better indicator of 'inhibition behaviour'.

The use of in-situ atomic force microspcopy allows us to monitor the crystal surface at high resolution as an additive is introduced into the supersaturated solution. Atomic-resolution images of growth features on crystal surfaces are now achievable (see Fig. 4), and thus we are seeking to combine modeling with AFM imaging and studies of bulk morphology changes in order to "close the loop" between additive design and crystal morphology control.

\section{Summary}

The examples described above demonstrate the variety of factors that come into play when considering the relative effects of crystal growth modifiers, even when these molecules contain similar structural units or interact with similar crystal systems. Through a combination of experiments and computer modeling we have found that the rational design of crystal growth modifiers to direct specific morphologies requires a detailed understanding of how additives interact with growth features at the crystal surface, rather than simply through interactions with flat crystal surfaces. Future work will aim to develop this approach in order take us closer to the goal of designing tailor-made additives to direct the morphology of nanoparticles for specific applications.

\section{References}

[1] M.-P. Pileni: Nature Materials Vol. 2 (2003), p. 145.

[2] F. Jones, A. Oliveira, A.L. Rohl, G.M. Parkinson, M.I. Ogden and M.M. Reyhani: J. Cryst. Growth Vol. 237-239 (2002), p. 424.

[3] F. Jones, J. Clegg, A. Oliveira, A.L. Rohl, M.I. Ogden, G.M. Parkinson, A.M. Fogg and M.M. Reyhani: Cryst. Eng. Comm. Vol. 40 (2001), p. 1.

[4] F. Jones, M.I. Ogden, A. Oliveira, G.M. Parkinsonand W.R. Richmond: Cryst. Eng. Comm. Vol. 5 (2003), p. 159.

[5] M.A. Nygren, D.H. Gay, C.R.A. Catlow, M.P. Wilson and A.L. Rohl: J. Chem. Soc. Faraday Trans. Vol. 94(1998), p. 3685. 